\title{
Research on the Application of Chinese Characters in Art Design
}

\author{
Chengzhi $\mathrm{Yu}^{\mathrm{a}}$, Yicheng Zhang ${ }^{\mathrm{b}}$
}

School of Jingdezhen Ceramic University College of Science and Technology, Jiangxi, 333000, China

a376597000@qq.com, b502684043@qq.com

Keywords: Chinese character; art design; application.

\begin{abstract}
The design of Chinese characters is very important in the art design. Clever use of creative expression of Chinese characters font design is a method to show the brand image of modern art design. The graphic feature of the text makes the design of font Chinese characters more artistic, being skillfully used in a variety of art designs. This article demonstrates the application effect in the art design of the font design Chinese characters and the future development trend.
\end{abstract}

\section{Introduction}

The composition and design of the text is a kind of structure form which is created by the "form" of Chinese characters. The modern graphic feature of the text is not only a hieroglyph recorded, but integrating more human emotion and aesthetic charm to express the willing of the people. Modern graphic feature of the text focuses on visual effect on representation, form, expression, richness, and aesthetic these five aspects.

\section{Basic Principles of Chinese Character Design}

As an abstract element, the design of Chinese theme characters itself is composed of points, lines and planes, and they can also form a new point, line and surface. In modern art design, the composition representation of point, line, and surface will make the Chinese characters font design with obvious content and tone [1, 2], and it is easy to feel the emotions that the font design Chinese characters expected to express, achieving the role of emotional communication bridge between packaging and consumers, and also the re-creation of visual aesthetic.

As shown in Figure 1 is the Chinese character font design of "Zhonghua", which has a good visual effect, easy to identify and memorize [3].

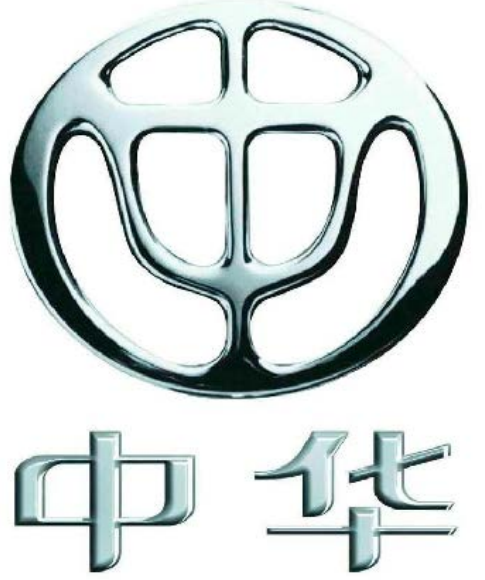

Figure 1. Chinese character design of "Zhonghua"

As shown in Figure 2 "Jing", the "Chinese seal" logo design in the 2008 Beijing Olympic Games chose "Jing" as a symbol of the text graphics, which expresses the image of a person in the running and highlights the Olympic sports health theme; at the same time through this font design Chinese characters, it is known that the 2008 Olympic Games was held out in Beijing; in addition, the character "Jing" is designed as a symbol like the text of "Wen", highlighting the time theme of 
"Humanistic Olympics" [4]. The text of the modern image fuses the "Beijing Olympics", "humanities" and "sports theme" these three themes information at the same time, and display in the form of the most profound ancient art of Chinese seal, which not only highlights the time theme of the Chinese characters graphical feature, and display Chinese ancient culture and art to the world, to spread the Chinese culture. Figure 2 shows "Chinese seal" logo design in the Beijing Olympic Games [5].

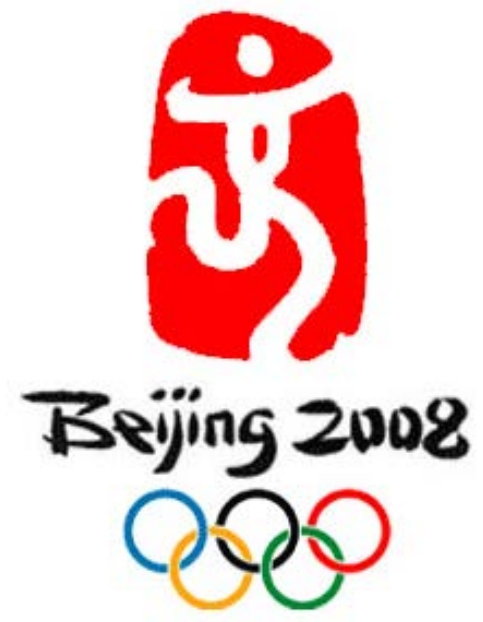

Figure 2. The logo design of "Chinese seal" in Beijing Olympic Games

\section{Relationship between Chinese Character Design and Art Design}

\subsection{Relationship between Chinese Characters Orientation and the Image Strategy of Art Design}

The most prominent feature of the application of Chinese character font design in the art design is that it is easy to be identified, which fully reflects the product information, visualization, artistic and other visual language features. The application of the font design of Chinese characters in art design is the integrated and concise summary of enterprise culture and brand image. No matter what the Chinese characters font design in art design expresses or indicates, whether it is moral or symbolic meaning, the meaning and enterprise culture should be unified.

In art design, we can use the font design of Chinese characters as interpretation of the commodity properties of media, processing graphic by changing the font shape itself and font structure; also can be reasonably used and transferred from space layout relations, that is, regard words as the basic point, line and surface elements to carry out the layout design, thus making it have a strong form aesthetic [6]. But no matter what kind of forms, they are to find usable graphic elements from the text itself, and according to the commodity attribute to re-design the words with graphic feature, enhancing product visual performance and reflecting the product brand image style.

\subsection{Relationship between Chinese Character Design Orientation and Visual Image of Art Design}

Sometimes the subject text graphic occupies a large part even whole part in art design. The graphic design of the theme word itself can be achieved by symbolic or allegoric techniques to add or subtract strokes, add decoration or change the font structure to expand the image, which emphasizes the profound meaning and visual effects that the font expresses.

\section{Application of Chinese Character Design in Art Design}

\subsection{Use of Chinese Character Design in Packaging Design}

From the concept, the formation of the surface is generated by the trajectory of the line. The different segmentations and layouts of surface will produce different visual effects, which can not only generate ease feeling, but also form a sense of oppression, reflecting different visual movements. The existence of theme text makes the entire packaging layout very vivid and full. When composing 
of the surface, overall plan the relationship between the elements and the harmony of pictures. When necessary, it can even make some blank treatment in packaging; in three-dimensional packaging, it can also make a crossover on two planes for the text arrangement, so as enhance the visual tension and artistic effect. Chinese characters font design method are mainly divided into the following several parts:

(1) Display in calligraphy text

The calligraphy ink has a flow direction, the writing process itself that cannot be repeated is a kind of graphical representation, and also a representation method of time to drive space and the action process to drive visual. For example, tea art design in Figure 3 is to present in the text of the ancient calligraphy, in which China text charm fully reflects the green charm of tea. Art design is the first impression of products putting on the market. The design of tea packaging in Figure 3 gives the new initial impression, making the good sense for consumer. Figure 3 shows the packaging design for a tea [7].

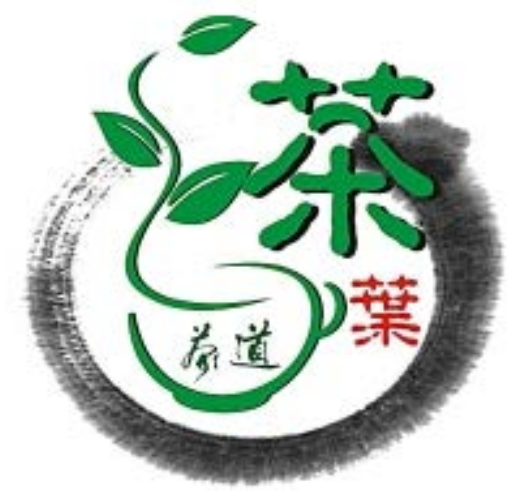

(2) Split text strokes

Figure 3. Packaging design of tea

Split the strokes of one or more Chinese characters and reconvict the structure, then the identification of text is reduced, but the graphical feature is enhanced, generally requiring comments. After splitting, the re- combining text loses the function of straightforward interpretation, but new graphic form has a more profound meaning. As shown in Figure 4 [8], the moon cake packaging, the two words "Hui Jian" are combined and skillfully transformed, fully embodying the best wishes of going home to reunion the family members in Mid-Autumn Festival, packaging vividly and iconically conveying the main idea of products.

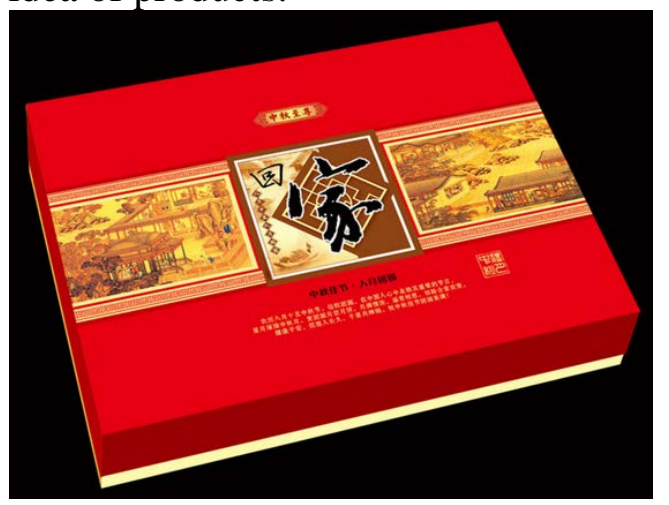

Figure 4."Hui Jia" moon cake packaging design

(3) Reserve the text blank

The application of Chinese characters in print advertising design can display the highlights of the product, which is reflected in the differences in the creative aspects of the elements of the plane advertisement. The application shape, size, color, depth and divergence of the Chinese characters in the design of print ads, the combination can bring people with different visual and spiritual feelings and artistic charm in different levels. In the past few years, the representation form of the Chinese character font in the design art of commercial advertising has obtained a multi-angle visual effect, revealing the style of the times. 
As shown in Figure 5, the packaging design of "Hua kai fu gui", fresh and simple font and concise surrounding space leave consumers with a proper imagination space. In the three-dimensional packaging, it can also apply the text layout in the crossover of the packaging and products and strengthen the link between packaging and products. Select the fan Chinese characters font adornment that has the ancient charm. In the external package, butterflies and peony full of vigor and clearly reflect the elegance of the gift, and in the use of "Hua kai fu gui" [9] these four words, use different fonts, words of different sizes with outstanding features brand, the packaging design using text graphic, in addition to retaining the blossoming text marks, red peony and butterfly and a small amount of commodity information, almost no longer use any decorative patterns. The overall effect is simple and bright packaging, achieving visual effects and psychological effects and conveying a calm attitude of a long history and taste of life. This design full of text graphic makes the entire packaging more prominently showing brand image, successfully achieving a balanced consideration of its function and emotional appeal.

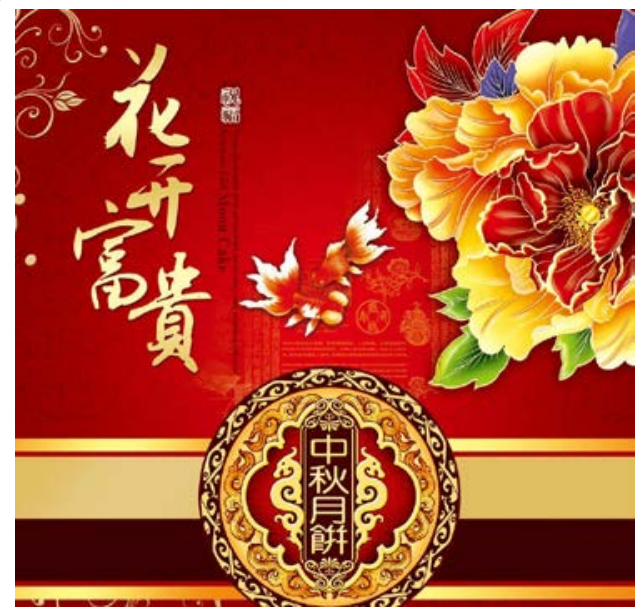

Figure 5. The packaging design of "the flower is rich"

\subsection{Application of Chinese Characters in Web Page Design}

Chinese font, as the elements of a web page design, directly impacts the overall image of the web page. The use of Chinese characters in web design, as a network information dissemination platform, enriches people's lives, and brings the visual effects and psychological effects different from the traditional media. Shade change in the official script and italics, bold and Arial form colorful color layers; font lines for false and true, the font size and coarse light can be adjusted freely so that the homepage art design produces distant and open-minded artistic conception, thus integrating the artistic conception of Chinese characters in new media communication.

\subsection{Application of Chinese Characters in the Books Design}

The Chinese characters font is used in the book in the form of mark, embellishment, and illustration, bringing the effect of total new, which plays the role of spreading the font Chinese characters culture. No matter from the decorative design or art design application, it has inherited the smart line charm that Chinese characters font characteristics represented. Chinese characters illustrations in the book create a cultural and active atmosphere so that the book is full of vitality. For example, the book "Xing huo liao yuan" chose Mao Zedong cursives, grand and magnificent, elegant and easy, without using other elements. Only these four words have highlighted the book layout design features. In addition, some books use a calligrapher calligraphy to write the title, because the popularity of the calligrapher and his understanding and creation of books enhance the book's artistic personality; some collocate calligraphy font with book content, composition, pattern and so on elements, and find new inspiration in the edge relation to integrate with subjective thoughts, thus the book binding effect is more infectious. The art design of "Xing huo liao yuan" cover is shown in Figure 6 [10]. 


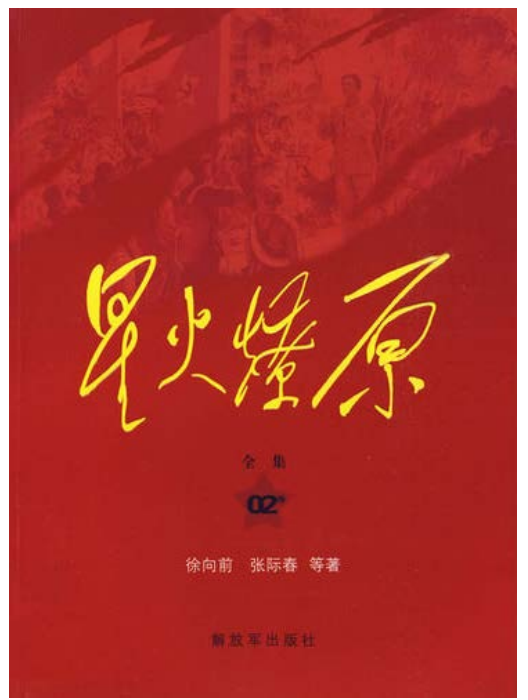

Figure 6."Xing huo liao yuan" cover art design

\subsection{Application of Chinese Characters in the Album Cover Design}

According to the Chinese characters font, extract various patterns, and through the combination of lines, they can be used to China card design. For instance, the design in Figure 7 [11], it not only refers to the quotation of characters' font record card, but also the inheritance of Chinese cultural and artistic spirit. With the rapid development of music and computer art design, Chinese characters font culture began to spread in the faster and newer channel in the form of Chinese characters font record card, so the font Chinese characters art and modern graphic design are connected, to realize the harmonious co-existence of music and painting. Thus, record appearance has strong ethnic flavor, and do not lose fashionable breath, which not only reflects the music theme of records, but also the album covers full of cultural heritage. Figure 7 is the art design of the record covers.

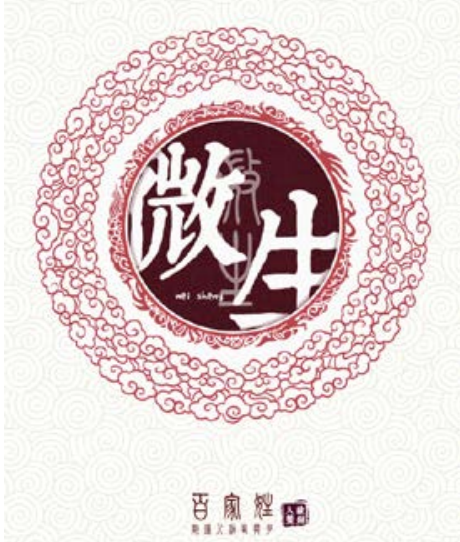

Figure 7. Art design of record cover?

\section{Conclusion}

With the development of society and the change of economic mechanisms and life festival accelerated, people expect the information communication and exchange more convenient, fast, and intuitive. Based on this demand, the graphic design of the text gets faster inheritance in art design, and the application of text design in art design starts tending to be simplified, abstract and symbolized. Especially in the art design, the text design can be more concise, intuitive, and iconical expression of product information and product content [12]. Consumers have a preliminary cognition and memory of products through words, font art design of theme Chinese characters is the image display of enterprise brand, and the key factor to improve the image of the product, the visual link connected the 
consumer and the product. Reasonable and scientific font design of theme Chinese characters plays an important role in the market generalization and marketing of the product, marketing. Font design of theme Chinese characters, as a visual element of modern art design, in the application process, in addition to clearly express product information, but also should be integrated with the aesthetic concept and cultural connotation and continuously innovate, so as to meet the consumers' visual aesthetic effect and psychological effect on emotional demands of products, making the product image being deep the heart and enduring fragrance.

\section{References}

[1] Tao D, Lin X, Jin L, et al. Principal component 2-D long short-term memory for font recognition on single Chinese characters [J]. IEEE transactions on cybernetics, 2016, 46(3): 756-765.

[2] Chen Z, Rau P L P, Chen C. How to design finger input of Chinese characters: A literature review [J]. International Journal of Industrial Ergonomics, 2014, 44(3): 428-435.

[3] Sze W P, Liow S J R, Yap M J. The Chinese Lexicon Project: A repository of lexical decision behavioral responses for 2,500 Chinese characters [J]. Behavior Research Methods, 2014, 46(1): 263-273.

[4] Zhen Z. Advantages and Thinking on Design of Chinese Characters' Graphics [J]. Studies in Literature and Language, 2015, 11(2): 82-87.

[5] Xu M, Kao H S R, Zhang M, et al. Cognitive-neural effects of brush writing of Chinese characters: cortical excitation of theta rhythm [J]. Evidence-Based Complementary and Alternative Medicine, 2013, 2013.

[6] Yu L, Reichle E D, Jones M, et al. RadicalLocator: A software tool for identifying the radicals in Chinese characters [J]. Behavior research methods, 2015, 47(3): 826-836.

[7] Luo C, Dell' Acqua R, Proctor R W, et al. Lack of visual field asymmetries for spatial cueing in reading parafoveal Chinese characters [J]. Psychonomic bulletin \& review, 2015, 22(6): 1764-1769.

[8] Tang W W W, Leong H V, Ngai G, et al. Detecting handwriting errors with visual feedback in early childhood for Chinese characters[C]//Proceedings of the 2014 conference on Interaction design and children. ACM, 2014: 273-276.

[9] Lam H C, Tsui B M A. Drawing on the variation theory to enhance students' learning of Chinese characters [J]. Instructional Science, 2013, 41(5): 955-974.

[10] Wu C Y. Generation of landmark architecture and sculpture based on Chinese characters: U.S. Patent 8, 836, 699 [P]. 2014-9-16.

[11] Su L, Zeng Z. Discussion on the First Lesson in Chinese Characters Teaching to the Foreign Students [J]. Theory and Practice in Language Studies, 2015, 5(12): 2545.

[12] Huang T, Zhang S, Duan X, et al. On-Line Handy Handwriting Chinese Characters Input for Non-Chinese Speakers Based on Wavelet Neural Network [J]. International Journal of Pattern Recognition and Artificial Intelligence, 2016, 30(06): 1659017. 